\title{
Freezing by Heating in a Driven Mesoscopic System
}

\author{
Dirk Helbing, ${ }^{1,2, *}$ Illés J. Farkas, ${ }^{2, \dagger}$ and Tamás Vicsek ${ }^{2, \ddagger}$ \\ ${ }^{1}$ II. Institute of Theoretical Physics, University of Stuttgart, Pfaffenwaldring 57/III, 70550 Stuttgart, Germany \\ ${ }^{2}$ Department of Biological Physics, Eötvös University, Budapest, Pázmány Péter Sétány 1A, H-1117 Hungary
}

(Received 22 April 1999)

\begin{abstract}
We investigate a simple model corresponding to particles driven in opposite directions and interacting via a repulsive potential. The particles move off-lattice on a periodic strip and are subject to random forces as well. We show that this model-which can be considered as a continuum version of some driven diffusive systems - exhibits a paradoxical, new kind of transition called here "freezing by heating." One interesting feature of this transition is that a crystallized state with a higher total energy is obtained from a fluid state by increasing the amount of fluctuations.
\end{abstract}

PACS numbers: 64.60.Cn, 05.70.Fh, 05.70.Ln

Most of the phenomena in our natural environment occur under far-from equilibrium conditions resulting in a rich behavior both in time and in space. An important class of such processes takes place in so-called driven systems, which have attracted considerable interest recently. In many of these systems, particles either are driven by an external field (force) [1-3] or are self-propelled [4,5], and their collective behavior manifests itself in new kinds of transitions, including noise-induced ordering [2] or ordering in a continuous $2 \mathrm{D}$ velocity space [4].

Phase transitions are also common in equilibrium systems, and the related analogies have represented an important contribution to the understanding of nonequilibrium processes. In most cases, lattice models have been considered to demonstrate nonequilibrium transitions. For example, jamming transitions have been seen in discretized traffic models [7] and driven lattice gases [8]. However, off-lattice (i.e., continuum) symmetry is known to bring in qualitatively new behavior; in particular, this is definitely so in 2D, see, e.g., the $X Y$ model [9] versus the Ising model (in equilibrium). A continuum model may lead to new effects due to the fact that the notions of order and disorder have extra facets in this case, and it can describe compressible systems in a more delicate way.

In this paper we will consider a simple continuum model exhibiting a paradoxical, new kind of transition that we call "freezing by heating" and being closely related to situations relevant from the practical point of view. The model consists of particles driven in opposite directions and interacting through a simple repulsive potential. The particles move off-lattice on a periodic strip (in a two-dimensional tube) and are subject to random forces as well. The most interesting feature of the transition we find for this system is that a crystallized state with a higher total energy is achieved from a fluid state over a transient disordered state by increasing the amount of fluctuations.

In addition to the interest in the properties of driven systems on its own, there are several further motivations to study such a model. A system of light (rising) and heavy (sinking) particles in a vertical column of fluid, pedestrians moving in a passage, or a system of oppositely charged colloidal particles in an electric field represent potential applications of our model. In fact, the system we study is a generalization to the continuum case of a two-species driven lattice gas model proposed recently [10], with a number of relevant modifications arising from the adaptation to the off-lattice case. In a wider context, these models can be considered as simplified paradigms of systems consisting of entities with opposing interests (drives). In the present work, we consider the behavior of a limited number of particles in a confined geometry, and our results are primarily valid for this "mesoscopic" situation. In the quickly growing literature on mesoscopic systems there are many examples of the potential practical relevance of phenomena occurring in various models for finite sizes [11].

We denote the location of particle $i$ at time $t$ by $\boldsymbol{x}_{i}(t)$ and its velocity $d \boldsymbol{x}_{i}(t) / d t$ by $\boldsymbol{v}_{i}(t)$. Furthermore, we assume the acceleration equation

$$
\begin{aligned}
m \frac{d v_{i}(t)}{d t}= & m \frac{v_{0} e_{i}-v_{i}(t)}{\tau}+\xi_{i}(t) \\
& +\sum_{j(\neq i)} f_{i j}\left[x_{i}(t), x_{j}(t)\right]+\boldsymbol{f}_{\mathrm{b}}\left[\boldsymbol{x}_{i}(t)\right] .
\end{aligned}
$$

$m$ is the mass of the particle, $v_{0}$ the velocity with which it tends to move in the absence of interactions, $\tau$ the corresponding relaxation time, and $\boldsymbol{e}_{i} \in\{(1,0),(-1,0)\}$, the direction into which particle $i$ is driven. $\gamma=m / \tau$ may be interpreted as a friction coefficient. $f_{i j}$ represents the repulsive interactions between particles $i$ and $j, f_{\mathrm{b}}$ the interactions with the boundaries, and $\xi_{i}$ the fluctuations of the individual velocities. For the interactions between the particles, we have chosen the simple function

$$
\boldsymbol{f}_{i j}\left(\boldsymbol{x}_{i}, \boldsymbol{x}_{j}\right)=-\nabla A\left(d_{i j}-D\right)^{-B},
$$

depending on the parameters $A$ and $B$, and the distance $d_{i j}(t)=\left\|\boldsymbol{x}_{i}(t)-\boldsymbol{x}_{j}(t)\right\|>D$ only. Thus, $\boldsymbol{f}_{i j}$ describes the effect of a soft repulsive potential of particle $j$ with a hard core of diameter $D$, reflecting the space occupied 
by the particle. Our choice of these details of the model corresponds to a motion of finite sized particles tending to avoid collisions and maintaining, if possible, a givenvelocity $v_{0}$. In addition, the interactions with the boundaries were assumed to be

$$
\boldsymbol{f}_{\mathrm{b}}\left(\boldsymbol{x}_{i}\right)=-\nabla A\left(d_{i \perp}-D / 2\right)^{-B},
$$

where $d_{i \perp}$ denotes the shortest distance to the closest wall.

In contrast to previous studies of similar models [12], we will now investigate the decisive role of the fluctuations $\boldsymbol{\xi}_{i}(t)$, which have been assumed to be uncorrelated and distributed according to a truncated normal distribution with vanishing mean value and finite variance $\theta$ [13]. We started our simulations with $N$ particles randomly distributed on a strip without allowing overlaps. For half of the particles a driving into the $(-1,0)$ direction and for the other half a driving into the $(1,0)$ direction was assigned. Numerical integration of Eq. (1), using periodic boundary conditions, has produced the following results: For small noise amplitudes $\theta$ and sufficiently small particle densities, our simulations lead, depending on the strip width and the initial condition, to the formation of two or more coherently moving linear structures (just as if the particles moved along traffic lanes) (cf. Fig. 1a). For relatively large $N$ (if the available area is too small to allow freely moving lanes), jamming occurs. For a small intermediate density region, we find lane formation or jamming, depending on the respective initial condition.

At small noise amplitudes, the mechanism of lane formation, which produces a "fluid" state, is very dominant and robust in our model. This can be understood as follows: Particles moving against the stream or in areas of mixed directions of motion will have frequent and strong interactions, because of high relative velocities. In

(a)

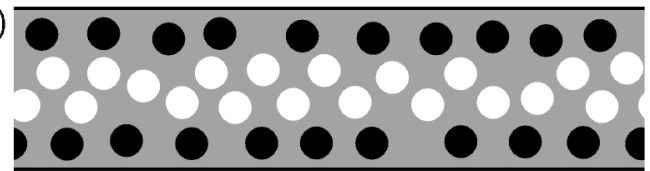

(b)

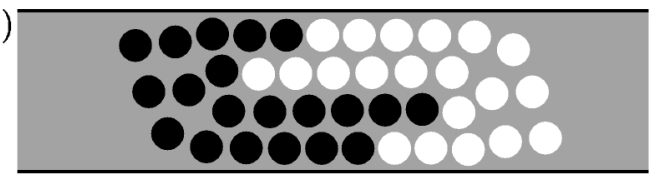

(c)

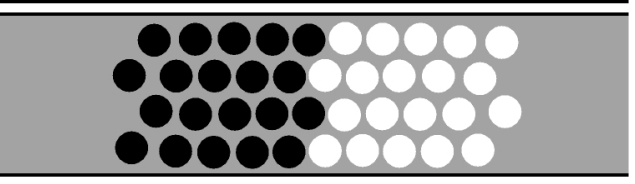

FIG. 1. Simulation of 20 particles moving from left to right (black) which interact with 20 particles moving from right to left (white) on a periodic strip of length $L_{x}=20$ and width $L_{y}=5$ at different noise intensities. The model parameters are $m=1$, $D=1, v_{0}=1, A=0.2, B=2$, and $\tau=0.2$. (a) Lanes of uniform directions of motion forming at small noise intensity $(\theta=1)$. (b) Snapshot of an intermediate jammed state with a rough interface, which is about to form "channels." (c) Final crystallized state resulting for large noise intensity $(\theta=1000)$. each interaction, the encountering particles move a little aside to pass each other. This sidewards movement tends to separate oppositely moving particles. Nevertheless, jamming may sometimes occur, but in most cases it also supports lane formation (see next paragraph). Particles moving in uniform lanes have very rare and weak interactions. Hence, the tendency to break up existing lanes is negligible, when the fluctuations are small. Furthermore, the most stable configuration corresponds to a state with a minimal interaction rate and is related with a maximum efficiency of motion [14].

Whereas spontaneous lane formation was also observed in previous studies of related models with deterministic dynamics only [12], in the present, more realistic model we have discovered a surprising phenomenon when we increased the noise amplitude. If the fluctuations and the particle number are large enough, the particles crystallize into a hexagonal lattice. This is a consequence of several subsequent steps: First, the fluctuations are able to prevent lane formation or even to destroy previously existing lanes. This is so because sufficiently strong diffusion can prevent structure formation. Second, some of the oppositely moving particles block each other locally from time to time. Third, this gives rise to jamming since, meanwhile, additional particles arrive at the boundaries of the blocked area. Fourth, if the jam exists long enough, both of its ends expand over the full width of the strip and develop "flat" boundaries perpendicular to $e_{i}$, in order to reach a balance of forces. For the same reason, the particles tend to arrange in a hexagonal lattice structure, very much like in a crystal. Fifth, the crystal is only stationary, if also the interface between the oppositely moving particles is, by chance, flat enough (cf. Fig. 1c). In most cases, however, the interface is rough (cf. Fig. 1b); i.e., in some of the horizontal layers, a majority of particles is pushing in one direction. As a consequence, the most advanced part(s) of the interface eventually break(s) through, which requires a continuous model, where the distance kept among the particles is flexible enough. In this way, particles with uniform directions of motion form "channels," which tend to produce lanes at sufficiently small densities and noise intensities; otherwise the particles jam again and again (as described above), until they end up in a stationary crystal.

Because of the above described mechanism the crystallized state is metastable, i.e., sensitive to structural perturbations (like the interchange of a few particles in our case). The crystallized state can also be destroyed by ongoing fluctuations with extreme noise amplitudes giving rise to a third, disordered ("gaseous") state with randomly distributed particles. Thus, with increasing "temperature" $\theta$, we have the untypical sequence of transitions fluid $\rightarrow$ solid $\rightarrow$ gaseous.

Interestingly, for a range of moderate densities we find a fluid state with lanes at small noise amplitudes most of the time, but a crystallized ("frozen") state, if the noise amplitude (temperature) is large. We call this transition freezing 
by heating. Starting with random initial conditions, the transition is rather smooth (cf. Fig. 2). This is partially so because the system can also become frozen at a relatively low noise amplitude, if the disorder in the initial state (in the sense of the deviation from a freely moving lane state) is large enough (which has an effect similar to additional fluctuations). The transition becomes sharper, if we always start with a two-lane state but with different random seeds. In any case, the transition is hysteretic, since the noiseinduced frozen state remains, when the noise amplitude is reduced, again.

To characterize the state of the system, we calculated various quantities. The expression

$$
E=\lim _{T \rightarrow \infty} \frac{1}{T} \int_{0}^{T} d t \frac{1}{N} \sum_{i=1}^{N} \frac{\boldsymbol{v}_{i}(t) \cdot \boldsymbol{e}_{i}}{\boldsymbol{v}_{0}},
$$

for which we expect the relation $0 \leq E \leq 1$, is a measure for the "efficiency" of motion; i.e., $E v_{0}$ is the average speed at which the particles are able to move in their respective "target direction" $\boldsymbol{e}_{i} . E \approx 1$ corresponds to lanes, $E=0$ to a crystallized state. Representative simulation results for the ensemble average $\langle E\rangle$ as a function of the noise intensity $\theta$ are displayed in Fig. 2.

We observed the following parameter dependencies: Crystallization is more pronounced for large $\tau$ and large strip lengths $L_{x}$, while small $\tau$ and large strip widths $L_{y}$ are in favor of lane formation (but freezing by heating still exists in the overdamped limit $\tau \rightarrow 0$ ). The number of particles required for crystallization does not depend

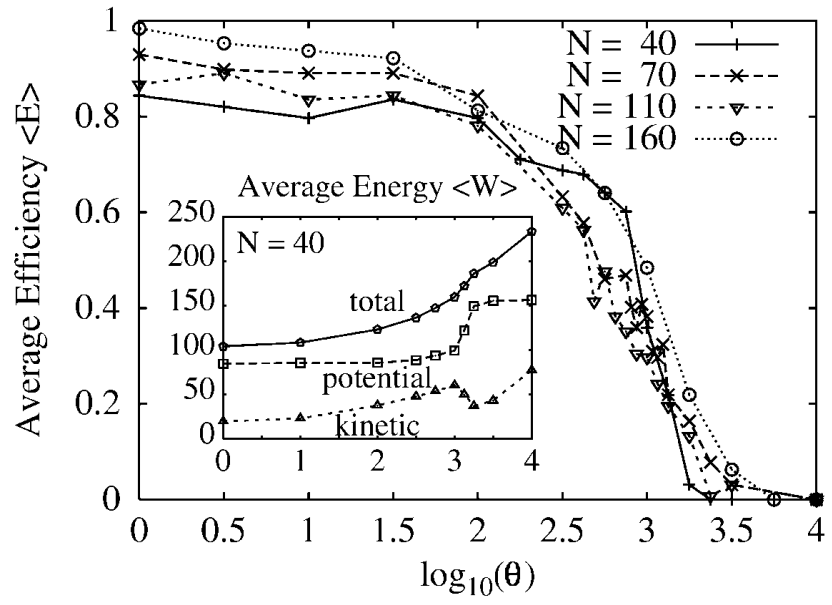

FIG. 2. Efficiency $\langle E\rangle$ of the system as a function of the noise intensity $\theta$ for various system sizes with a fixed aspect ratio of $L_{x} / L_{y}=4: 1$ and the parameter values displayed in Fig. 1. Shown are averages over 128 (for $N=160$ : 64) simulation runs with different random seeds. The inset shows the average potential, kinetic, and total energy in the resulting system states. While the potential energy (- - ) increases with the noise amplitude due to jamming, the kinetic energy (- - -) is composed of a contribution proportional to $\theta$ and a decreasing contribution $N m\left(v_{0}\langle E\rangle\right)^{2} / 2$. The total energy (-) turns out to be an increasing function of $\theta$. on the length $L_{x}$ (if it is considerably larger than $L_{y}$ ), while it is roughly proportional to the width $L_{y}$ (for large enough $L_{y}$ ). Given a fixed aspect ratio $L_{x} / L_{y}$, for the system sizes that we could numerically handle there was no clear tendency whether the transition becomes sharper or smoother with increasing system size $N \propto L_{x} L_{y}$ (see Fig. 2).

Another interesting quantity is the sum of the potential and kinetic energies associated with a given state:

$W=\lim _{T \rightarrow \infty} \frac{1}{T} \int_{0}^{T} d t\left[\sum_{i} \frac{m}{2} \boldsymbol{v}_{i}^{2}+\frac{1}{2} \sum_{i \neq j} A\left(d_{i j}-D\right)^{-B}\right]$.

The paradox here is that the above mentioned crystallized state is usually more unstable than the fluid state, in the sense that the total energy (5) of the system is higher in the crystallized state than in the fluid one (see inset of Fig. 2). Note that both the "solid" (crystallized) state and the fluid state (i.e., lanes) are destabilized, if the friction term $-\boldsymbol{v}_{i} / \tau$ is dropped, even in the case $\theta=0$ (see Ref. [15] for a related inverse phenomenon). That is, without friction and due to the permanent driving, the undamped repulsive interactions eventually become destructive to any ordered state, which gives rise to a gaseous state. Therefore, we point out that the energetically less favorable crystallized state is maintained by the propulsion term $m\left(\boldsymbol{v}_{0} \boldsymbol{e}_{i}-\boldsymbol{v}_{i}\right) / \tau$ in Eq. (1) which, by the way, is also relevant for lane formation. Note that the absolute value of this term becomes largest for $\boldsymbol{v}_{i}=\mathbf{0}$ (i.e., blocking), while it is small for the fluid state with $\boldsymbol{v}_{i} \approx \boldsymbol{v}_{0} \boldsymbol{e}_{i}$.

We consider the transition to a stationary state with a higher total energy by increasing the noise intensity to be a signature of a novel class of behavior in certain nonequilibrium systems, which may have interesting applications. However, here we have demonstrated freezing by heating only for limited sizes and a specific geometry. Nevertheless, we point out that freezing by heating does not require walls, but occurs for periodic boundary conditions in $y$ direction as well. Also, we do not need periodic boundary conditions in $x$ direction. Any sufficiently long simulation area will produce both the organized fluid state (i.e., lanes) and the crystallized state, if only the system is continuously entered by particles at the left-hand and right-hand boundaries.

Why is freezing by heating new? Some glasses may crystallize when slowly heated. However, this is a well understood phenomenon: the amorphous state is metastable for those temperatures, and crystallization means an approach to the more stable state with smaller total energy. In general, one can distinguish three cases when fluctuations (i.e., temperature or external perturbations) are increased: (i) Total energy increases and order is destroyed (e.g., melting). (ii) Total energy decreases, ordering takes place, and the system goes from a disordered metastable to an ordered stable state (e.g., in metallic glasses and some 
granular systems). (iii) Total energy increases and ordering takes place, while the system goes from a partially ordered stable to a highly ordered metastable state, which corresponds to the new situation presented here.

In our case, crystallization is achieved by spontaneously driving the system with the help of noise uphill towards higher total energy. The system would like to maximize its efficiency [14], but instead it ends up with minimal efficiency due to noise-induced crystallization. The role of temperature or noise here is to destroy the energetically more favorable fluid state, which inevitably leads to jamming and finally to crystal-like lattices. The corresponding transition seems to be related to the off-lattice nature of our model and is different from those reported for driven diffusive systems on a lattice $[8,10]$. It should be noted that the transition we find is not sharp, which is a consequence partly of the mesoscopic nature of the phenomenon and partly of the disorder in the initial state.

We point out that freezing by heating is likely to be relevant to situations involving pedestrians under extreme conditions (panics). Imagine a very smoky situation, caused by a fire, in which people do not know which is the right way to escape. When panicking, people will just try to get ahead, with a reduced tendency to follow a certain direction. Thus, fluctuations will be very large, which can lead to fatal blockings.

Our results demonstrate that in driven mesoscopic systems phenomena qualitatively different from those occurring in thermodynamical systems can be observed. While most nonequilibrium transitions have analogies to equilibrium ones [16], the noise-induced ordering observed in the effect of freezing by heating is just opposite to the transitions occurring in equilibrium systems. This suggests that future studies along the lines of the present approach are likely to lead to further unexpected findings.

D. H. wants to thank the DFG for financial support and D. Mukamel for helpful suggestions. This work was supported by OTKA F019299 and FKFP 0203/1997.

*Electronic address: helbing@theo2.physik.uni-stuttgart.de †Electronic address: fij@elte.hu

†Electronic address: vicsek@angel.elte.hu

[1] H. J. Herrmann, in Proceedings of the 3rd Granada Lectures in Computational Physics, edited by P. L. Garrido and J. Marro (Springer, Heidelberg, 1995); K. L. Schick and
A. A. Verveen, Nature (London) 251, 599 (1974); H. A. Makse et al., Nature (London) 386, 379 (1997).

[2] A. D. Rosato et al., Phys. Rev. Lett. 58, 1038 (1987); J. A. C. Gallas et al., Phys. Rev. Lett. 69, 1371 (1992); P. B. Umbanhowar et al., Nature (London) 382, 793 (1996).

[3] M. C. Cross and P.C. Hohenberg, Rev. Mod. Phys. 65, 851 (1993); P. Manneville, Dissipative Structures and Weak Turbulence (Academic Press, New York, 1990).

[4] T. Vicsek et al., Phys. Rev. Lett. 75, 1226 (1995).

[5] Y. L. Duparcmeur et al., J. Phys. I (France) 5, 1119 (1995); E. V. Albano, Phys. Rev. Lett. 77, 2129 (1996); H. J. Bussemaker et al., Phys. Rev. Lett. 78, 5018 (1997); J. Toner and Y. Tu, Phys. Rev. Lett. 75, 4326 (1995); E. Ben-Jacob et al., Nature (London) 368, 46 (1994); D. A. Kessler and H. Levine, Phys. Rev. E 48, 4801 (1993); D. Helbing et al., Nature (London) 388, 47 (1997); F. Schweitzer et al., BioSystems 41, 153 (1997).

[6] J.S. Walker and C. A. Vause, Sci. Am. 256, No. 5, 90 (1987).

[7] O. Biham et al., Phys. Rev. A 46, R6124 (1992); K. Nagel and M. Schreckenberg, J. Phys. I (France) 2, 2221 (1992); J. Krug and P. A. Ferrari, J. Phys. A 29, L465 (1996); D. Helbing and B. A. Huberman, Nature (London) 396, 738 (1998); D. Helbing and M. Schreckenberg, Phys. Rev. E 59, R2505 (1999).

[8] M. R. Evans et al., Phys. Rev. Lett. 74, 208 (1995); G. Szabó and A. Szolnoki, Phys. Rev. A 41, R2235 (1990); B. Schmittmann and R. K. P. Zia, Phase Transitions and Critical Phenomena, edited by C. Domb and J. Lebowitz (Academic Press, New York, 1995), Vol. 17; Phys. Rep. 301, 45 (1998).

[9] J. M. Kosterlitz and D. J. Thouless, J. Phys. C 6, 1181 (1973).

[10] B. Schmittmann et al., Europhys. Lett. 19, 19 (1992).

[11] Y. Imry, Introduction to Mesoscopic Physics (Oxford University Press, Oxford, 1997); N. G. van Kampen, Stochastic Processes in Physics and Chemistry (North-Holland, Amsterdam, 1981); M-L. Tan and I. Goldhirsch, Phys. Rev. Lett. 81, 3022 (1998).

[12] D. Helbing and P. Molnár, Phys. Rev. E 51, 4282 (1995).

[13] We limited the fluctuations to a maximum of $\left\|\boldsymbol{\xi}_{i}\right\|=3 \sqrt{\theta}$. Truncation at larger fluctuation levels gave practically the same results, but at the cost of considerably decreased computational efficiency.

[14] D. Helbing and T. Vicsek, New J. Phys. 1, No. 13 (see http://www.njp.org/).

[15] S. J. Cornell et al., Phys. Rev. Lett. 81, 1142 (1998).

[16] Phase Transitions and Critical Phenomena, edited by C. Domb and M.S. Green (Academic Press, New York, 1972-1976), Vols. 1-6; Phase Transitions and Critical Phenomena, edited by C. Domb and J.L. Lebowitz (Academic Press, New York, 1983-1995), Vols. 7-17. 